Revue internationale P.M.E.

Économie et gestion de la petite et moyenne entreprise

\title{
Les PME dans le commerce extérieur français : d'une proximité régionale à une proximité de délai
}

\section{Georges Giraud}

Volume 4, numéro 2, 1991

URI : https://id.erudit.org/iderudit/1008065ar

DOI : https://doi.org/10.7202/1008065ar

Aller au sommaire du numéro

Éditeur(s)

Presses de l’Université du Québec

ISSN

0776-5436 (imprimé)

1918-9699 (numérique)

Découvrir la revue

Citer cet article

Giraud, G. (1991). Les PME dans le commerce extérieur français : d'une proximité régionale à une proximité de délai. Revue internationale P.M.E., 4(2), 113-135. https://doi.org/10.7202/1008065ar
Résumé de l'article

Généralement considérées comme introverties face aux échanges internationaux, les PME paraissent plus actives dans une approche régionale du commerce extérieur. Les échanges extérieurs des régions françaises subissent un effet de frontière contrasté. La proximité économique avec les pays partenaires intervient autant sinon plus que la position géographique des régions. Dans les régions où les échanges sont denses, les grandes entreprises sont les plus extraverties, mais les PME ne sont pas exclues des flux internationaux. La sous-traitance internationale de perfectionnement bénéficie aux PME de type industriel, souvent bien insérées dans le tissu économique régional. Aujourd'hui, la proximité de délai tend à supplanter la proximité de lieu. Plutôt que du statut frontalier, l'avenir des échanges extérieurs des régions dépend dès lors des réseaux de PME présents dans les districts industriels. 


\title{
Les PME dans le commerce extérieur français : d'une proximité régionale à une proximité de délai
}

\author{
Georges GIRAUD * \\ CERAS, Université de Reims, IUT de Troyes
}

\begin{abstract}
RÉSUMÉ
Généralement considérées comme introverties face aux échanges internationaux, les PME paraissent plus actives dans une approche régionale du commerce extérieur. Les échanges extérieurs des régions françaises subissent un effet de frontière contrasté. La proximité économique avec les pays partenaires intervient autant sinon plus que la position géographique des régions. Dans les régions où les échanges sont denses, les grandes entreprises sont les plus extraverties, mais les PME ne sont pas exclues des flux internationaux. La sous-traitance internationale de perfectionnement bénéficie aux PME de type industriel, souvent bien insérées dans le tissu économique régional. Aujourd'hui, la proximité de délai tend à supplanter la proximité de lieu. Plutôt que du statut frontalier, l'avenir des échanges extérieurs des régions dépend dès lors des réseaux de PME présents dans les districts industriels.
\end{abstract}

\section{ABSTRACT}

Small firms are generally missing in foreign trade but they are more active in an approach by regions. Foreign trade of French regions is subject to a contrasted frontier effect. Economical proximity with partner countries plays a part as great if not greater than geographical place of regions. In intense trade regions, large firms are the most turned toward international exchange. But small firms are also active on it. Small firms of industrial type make a profit on international subcontracting for improvement, because they are often inserted in networks. Now, delay nearness supplants space proximity. In future, foreign trade of regions will depend on networks in marshallian industrial districts rather than on frontier effect.

* Georges Giraud, docteur en Sciences Économiques, assistant à l'Institut Universitaire de Technologie de Troyes depuis 1984 ; chargé de cours à la Faculté de Sciences Économiques de l'Université de Reims de 1978 à 1983 ; thèse de Doctorat portant sur «la durée de vie du capital : durée d'usage des équipements, durée d'immobilisation des capitaux», soutenue en décembre 1985 à Reims ; affilié au Centre de Recherche d'Analyse de Système de l'Université de Reims Champagne Ardenne ; a publié des rapports d'expertise en Économie Régionale et Industrielle de 1981 à 1989 ; travaux de recherche sur le commerce international et les PME depuis 1990. Adresse : IUT, rue de Québec, BP 396, 10026 Troyes Cedex, France. 


\section{RESUMEN}

Aunque generalmente consideradas como introvertidas respecto a los intercambios internaciones, las PyME ofrecen un aspecto mucho más activo en un enfoque regional del comercio exterior. Los intercambios exteriores de las regiones francesas están sujetos a un efecto de frontera contrastado. La proximidad económica con los países objeto interviene tanto o más que la situación geográfica de las regiones. Así, en aquellas regiones donde se da una fuerte densidad de intercambios, las grandes empresas son las más extravertidas, pero las PyME no quedan excluidas de los flujos internacionales. La subcontrata internacional de perfeccionamiento beneficia a las PyME de tipo industrial, generalmente bien implantadas en el tejido económico regional. Hoy día, la brevedad de plazos tiende a suplantar la proximidad de lugares. Más que de su estatuto fronterizo, el futuro de los intercambios exteriores de las regiones depende en adelante de las redes de PyME presentes en los distritos industriales.

\section{Introduction}

L'activité des petites et moyennes entreprises dans le commerce extérieur français paraît d'une grande modestie. La condescendance de l'analyse économique sur ce sujet fait écho à la complaisance des praticiens de la politique économique. Le constat de carence est unanime, les PME sont absentes du commerce extérieur malgré de rares quoique brillantes exceptions. L'effet de taille semble l'élément déterminant comme barrière à l'entrée. Jugées inertes face aux aléas du marché international, les PME sont par ailleurs définies par leur flexibilité. La singularité de cette contradiction nous interpelle et mérite plus qu'une simple phrase même si l'encéphalogramme plat des PME dans les statistiques nationales du commerce extérieur incite au fatalisme. Une telle déclaration d'absence des PME nous paraît suspecte en ce qu'elle semble faire l'économie d'une approche fine du problème. Elle limite les échanges extérieurs au commerce entre les nations et confine les PME dans les régions. A contrario, nous formulons l'hypothèse selon laquelle les PME exercent un rôle actif dans le commerce extérieur. La présente étude constitue une tentative pour valider cette hypothèse.

L'analyse du commerce extérieur renvoie aux grands espaces, alors que l'étude des PME relève plutôt du bassin d'emploi (Guichard, 1987). La distance logique est donc grande entre le terroir et la planète, l'hypofirme et les flux mondiaux. L'approche régionale du commerce extérieur doit permettre de réduire ce contraste conceptuel. C'est pourquoi nous nous attachons à saisir, ici, le rôle des PME dans le commerce extérieur français, à l'aide de l'échelon 
intermédiaire que constituent les régions. La démarche, à notre sens nouvelle, n'est pas aisée, car les échanges extérieurs n'ont de signification qu'en rapport avec un cadre intérieur pertinent. L'espace économique national dispose de la cohérence nécessaire pour remplir cette condition. Mais l'espace économique régional ne possède pas cette caractéristique. Les échanges de marchandises liés à la concurrence et à la spécialisation internationales ne sont toutefois pas éthérés. Ainsi, le commerce extérieur des régions n'existe pas, mais il faut bien admettre que la dimension régionale de ce commerce est présente.

Les outils traditionnels d'investigation du commerce international ne sont pas opératoires pour appréhender cette dimension. Un solde des échanges, un taux de couverture ou de pénétration régionale n'ont, ici, pas de véritable signification, car ils sont trop dépendants d'un vision nationale de la division du travail. L'équilibre des échanges étant rarement bilatéral ou permanent pour une nation, la mesure de tels indicateurs au niveau régional devient très relative. Il y a des pays clients, des pays fournisseurs; des produits d'approvisionnement et des produits d'expédition. Le niveau des exportations et des importations de marchandises est en relation directe avec l'existence de secteurs d'activité performants et compétitifs, exposés à la concurrence internationale. Il est aussi fonction de la présence de secteurs abrités de cette concurrence. Les disparités régionales en la matière amplifient les écarts. Malgré ces limites, l'approche régionale du commerce extérieur justifie l'objectif de cet article : tester le rôle des PME dans le commerce extérieur français. L'importance et l'implantation des PME sont plus repérables dans le tissu productif régional. La dimension spatiale des entreprises est plus nette au niveau régional.

Au terme de cette série de restrictions, le domaine d'interrogation est réduit. C'est le prix d'une démarche que nous désirons rigoureuse, mais qui reste sans doute perfectible. La réduction du champ fait mieux ressortir la qualité et l'importance des questions en suspens. L'intensité des flux régionaux du commerce extérieur est-elle fonction de la taille des entreprises ? Le statut frontalier des régions exerce-t-il une influence déterminante sur les échanges extérieurs ? Existe-t-il encore un effet de proximité tangible entre les régions et les pays limitrophes ? Cet effet est-il modulé par la densité du tissu productif? Quelle peut être la place des PME dans ces relations de proximité ? Cette place est-elle uniforme quel que soit le type d'entreprise ? Autant de questions dont la formulation délimite notre problématique dans cette étude. Les réponses que nous tenterons d'apporter à cette série de questions s'appuient d'abord sur le repérage des formes d'insertion des PME dans les flux régionalisés du commerce extérieur. Nous aborderons ensuite les modifications de cette insertion régionale à la lumière des caractéristiques récentes des échanges extérieurs : sous-traitance, flexibilité. 


\section{Les PME : seconds rôles ou figurants du commerce extérieur?}

\subsection{PME : une homogénéité apparente}

La concomitance de grandes entreprises orientées vers le marché international et de PME souvent introverties influence de façon singulière les échanges extérieurs. On peut penser que l'aptitude à exporter des grosses sociétés est en partie liée à la présence de PME autour des activités concernées. Cette pratique de la soustraitance se développe principalement dans le périmètre d'implantation des gros donneurs d'ordre, bien que cela ne soit pas exclusif. Les formes d'activité des PME paraissent avoir une dimension spatiale plus limité que les grandes entreprises. De façon générale, l'opposition des petites aux grandes, des faibles aux fortes ou même des incompétentes aux performantes est tenace, renforcée par l'approche factuelle (Biales et Rolland 1989). Elle relève pourtant d'une vision bien manichéenne. La distinction entre PME de type artisanal et PME de type industriel est sans doute plus fructueuse, à tout le moins plus subtile.

La catégorie des PME semble regrouper tout et son contraire. On y trouve des entreprises innovatrices, d'autres employant un personnel qualifié, ou positionnées sur un segment technologique précis. Mais on y observe aussi des entreprises ayant recours à la sous-traitance en cascade de manière systématique, alors que d'autres emploient toutes les ressources de flexibilité de l'intérim ou assure la vivacité du marché des équipements professionnels d'occasion. Le contraste est trop grand. L'homogénéité de la catégorie des PME se délite. La distinction PME de type artisanal (PMA) et PME de type industriel (PMI) permet d'éviter ce schisme factuel. Le sigle PMI n'est pas employé ici dans le sens commun de PME de l'industrie (Bucaille et Costa de Beauregard, 1987). Il renvoie à la différence entre organisation artisanale et industrielle des entreprises, que celles-ci exercent leur activité dans l'industrie, les services ou même l'agriculture.

Dans une PME de type artisanal, l'organisation des activités reste largement informelle, les modes de communication interne et la division du travail constituent des sous-ensembles flous, de même que le système de rémunération. Le savoir-faire pratique et l'implication directe du personnel occupent une place prépondérante dans l'activité principale. L'intensité capitalistique est souvent faible. L'image de l'entreprise, son apparition sur le marché passent très fréquemment par son dirigeant.

Dans une PME de type industriel, le travail et la communication sont mieux codifiés. Les règles de fonctionnement des activités sont nettement plus explicites. Le capital fixe productif est omniprésent (y compris sous forme de crédit-bail), le personnel chargé de sa mise en œuvre est plus qualifié. Les différentes fonctions (productive, commerciale, financière,...) quoique étroitement liées, sont bien dis- 
tinctes. Le mode d'organisation est objectif en ce sens qu'il échappe en grande partie à la personnalité du (des) dirigeant(s) (Gollac, 1989).

La différence PMA/PMI est principalement fondée sur des considérations internes aux entreprises. Elle relève aussi de facteurs externes comme la stratégie de produits ou le comportement sur les marchés (Hy, Lassaut et Nicolas, 1989). Ainsi, en matière de recherche et développement (R\&D), la distinction se fait sur la fréquence des recours aux institutions publiques ou parapubliques (ANVAR, Centres de Transfert de Technologie). Les relations soutenues avec les universités sont aussi discriminantes pour les PMI. Pour les PMA, les rares efforts de R\&D, sont plutôt liés au rôle personnel du patron. Plus la taille est réduite, plus l'implication des dirigeants dans la R\&D est grande (Temple, 1986).

Si la validation logique de la différenciation PMA/PMI paraît aisée, la démarche devient ardue lorsqu'on tente une validation empirique. L'expérimentation, au sens scientifique, étant illusoire en économie, les faits sont souvent réduits à leurs substituts statistiques (Di Ruzza, 1988). Dans ces conditions, tester la typologie proposée relève de la mission impossible, car les banques de données sont pauvres en information afférente aux critères de distinction sus-évoqués ${ }^{1}$.

Pour la suite de cette étude, la distinction est fondée, faute de mieux, sur les effectifs. Si l'on s'accorde à considérer le seuil des grandes entreprises à partir de 500 salariés, nous pouvons définir les PMI comme étant les entreprises relevant de la tranche 50 à 499 salariés, alors que les PMA sont dans la tranche inférieure d'effectifs. Ce critère univoque est loin de refléter l'extrême diversité des PME. Faute d'informations complémentaires détaillées, nous devons nous contenter de cette règle de présentation. Cela n'est pas satisfaisant, puisque l'on sait l'influence des liaisons financières sur l'organisation et les performances des entreprises. À taille comparable, les filiales des groupes de sociétés sont toujours mieux placées que les entreprises indépendantes. Ce sont en particulier les petites PME des groupes (moins de 50 salariés) qui sont les plus avantagées (Beau, 1990).

Il peut paraître dérisoire de proposer une typologie en deux classes là où d'autres ont montré l'hétérogénéité et la complexité des PME; ce qui est simple est faux (Marchesnay, 1991). On observera toutefois que les critères avancés ici, pour la distinction entre type artisanal et type industriel se déclinent tous selon les caractéristiques bien déterminées pour définir le concept de PME (Julien, 1990). La taille, le mode de gestion, le degré de spécialisation, la forme

1. La vérification statistique de la différence PMA / PMI nécessiterait la mobilisation de moyens et l'investigation de champs plus conséquents que ceux utilisés ici. L'auteur a conscience que cette modestie de moyens pourrait annihiler la portée du présent article si ce dernier montrait des ambitions classificatoires affirmées. Le lecteur aura compris que la démarche reste simplement euristique. La typologie PMA / PMI est donc réfutable au sens poppérien. 
de la stratégie, le caractère des relations internes et le type de relations externes permettent de distinguer grandes entreprises et PME. Ces mêmes caractéristiques peuvent servir à différencier PMA et PMI. Proposée à titre exploratoire, la typologie PMA / PMI mérite sans doute d'être approfondie de façon critique, notamment pour mieux qualifier la différence entre PMI et grandes entreprises. L'objectif de cette étude est simplement d'utiliser la distinction PMA/PMI dans l'analyse des flux régionaux du commerce extérieur puisque les PME ont une dimension régionale avérée.

La région d'implantation de l'entreprise constitue $83 \%$ de la zone de chalandise des entreprises de moins de 20 salariés. Le reste de la France en représente $15 \%$. Seulement $3 \%$ du chiffre d'affaires est réalisé avec l'étranger. Plus la taille de l'entreprise augmente, plus elle est présente sur les marchés situés hors de sa région (Berthier et Savoye, 1990). Cette relation reste vérifiée au-delà des frontières : exporter est une question de taille (Bricout, 1991). Cela étant, si la taille des entreprises est un critère décisif pour l'engagement à l'exportation, elle n'a pas d'influence directe sur le degré d'exportation. Le comportement des firmes en la matière paraît bien tranché : les entreprises sont peu, voire non exportatrices, ou bien très exportatrices. Une fois acquis, ce comportement est rarement modifié, les changements d'état sont marginaux. L'inertie dépasse $80 \%$ pour toute les classes d'entreprises (pas, peu ou très exportatrices) (Banon et Delbreil, 1989).

L'ouverture d'un secteur d'activité sur l'extérieur est fonction de son degré de concentration. Dans les secteurs les plus concentrés, le taux d'exportation est élevé (près de $50 \%$ ). Dans ces mêmes secteurs très concentrés, la pénétration étrangère est aussi nette: les importations couvrent près de la moitié de la demande intérieure. La part des PME nationales est limitée par l'ampleur des importations. La dimension internationale du marché constitue une barrière à l'entrée (Amar et Crepon, 1990).

L'énoncé de ces principes généraux, désormais bien établis, n'exclut pas un rôle actif des PME dans les échanges extérieurs. Le positionnement sur une niche étroite permettant de distancer ses concurrents par une extrême spécialisation est une stratégie pratiquée avec succès par quelques-unes d'entre elles (Maliverney, 1991). Mais pour l'essentiel, les PME restent en arrière-plan des marchés extérieurs. Ni inactives, ni indifférentes, elles sont plutôt sous-traitantes et n'apparaissent donc pas comme opérateurs de premier rang sur les marchés.

La sous-traitance industrielle est un facteur d'assouplissement de l'appareil productif. Elle s'inscrit dans la logique d'externalisation développée par les grandes entreprises durant la décennie 1980. La sous-traitance de capacité permet d'amortir les aléas des carnets de commande. Elle fait réaliser des économies notables d'investissement pour des équipements utilisés sporadiquement. La sous-traitance de spécialité évite d'engager les ressources de l'entreprise 
dans des savoir-faire trop pointus pour être pleinement intégrés à l'activité principale. Les entreprises sont d'autant plus sous-traitantes qu'elles sont de taille modeste (Quelennec, 1987). Cette modestie révèle une difficulté à paraître sur les marchés. Elle n'implique en rien une incapacité à produire. Il en résulte que la trace des PME est plus repérable dans l'activité régionale que dans les échanges internationaux.

\subsection{Les PME de type industriel mieux placées à l'export qu'à l'import}

En matière de commerce extérieur, les PME de type industriel semblent se distinguer, tant des PME de type artisanal que des grandes entreprises. La différenciation des régions par type d'entreprises n'est pas très marquée. Les grandes entreprises sont légèrement plus implantées dans les bassins industriels du Nord, de l'Est et du lyonnais. Les régions Sud accueillent nettement des PME de type artisanal. L'implantation régionale des PME de type industriel semble homogène à une ou deux exceptions près.

Les principaux résultats de l'analyse des flux régionalisés du commerce extérieur en fonction du type d'entreprises sont présentés dans la figure $1^{2}$. La

2. Lecture de la figure 1 : la région Limousin (LIM) paraît peu impliquée dans les échanges extérieurs et peu spécialisée par taille d'entreprises. Elle se distingue des autres régions par des exportations et des importations très faibles (X1, M1), ainsi que par la modestie et la rareté relative de ses entreprises dans l'industrie, quelle que soit leur taille (TA1, TI1, GT1). Elle est située dans le nuage de points des modalités faibles. À l'opposé, la région Nord Pas De Calais (NPC) semble très extravertie, surtout du fait de ses importations (M3). Elle se différencie des autres régions par la densité de ses grandes entreprises (GT3) ; elle est située dans le nuage de points des modalités fortes. Plus généralement, ce graphique représente la répartition de la distribution statistique étudiée selon les deux premiers axes dégagés par une analyse factorielle des correspondances multiples. Les individus observés sont les régions. Les exportations et les importations par produits constituent les variables actives. Les données de taille des entreprises sont ici des variables supplémentaires. Par souci de lisibilité, les variables détaillées sont gommées (ex.: exportations agroalimentaires) ; seuls les barycentres de chaque catégorie de variables sont positionnés (ex. : exportations). L'axe 1 explique $26 \%$ de l'inertie totale des données. Il oriente les valeurs des modalités faibles vers les modalités fortes. L'axe 2 représente $12 \%$ de la dispersion du nuage de points. Il oppose les modalités fortes aux modalités moyennes. Le caractère hétérogène des variables (exportations, importations et tailles) a nécessité une transformation en classes pour éliminer l'effet des unités différentes de mesure. Apparaissent ainsi des différences entre les régions observées ou entre les variables d'exportation, d'importation ou de taille. Pour cela, la distribution de chaque variable a été séparée en trois ensembles. Chaque individu est classé dans un ensemble de modalités (faibles, moyennes ou fortes), selon qu'il possède une valeur faible, moyenne ou forte, comparativement aux autres individus, pour la variable considérée. 
représentation graphique de la répartition régionale des modalités d'échanges correspond en majeure partie à celle des tailles des entreprises. Pour une région, l'absence de spécialisation par taille correspond à une indifférence dans les échanges extérieurs.

L'intensité des échanges extérieurs augmente avec la taille des entreprises. Les échanges d'intensité élevée renvoient aux grandes entreprises et aux PMI, non aux PMA. Les PME de type artisanal semblent hors marché. Les régions où les effectifs occupés par les PMI sont importants paraissent avoir une insertion honorable dans les échanges extérieurs régionalisés. Cette remarque est surtout vérifiée pour les exportations, y compris en modalités moyennes. Enfin, si les régions spécialisées dans les grandes entreprises sont bien placées pour les exportations de forte densité, elles le sont encore mieux pour les importations.

Mais la sensibilité des échanges à la taille n'est pas la même selon leur sens. Les exportations d'importance découlent des grandes entreprises, mais aussi des PME de type industriel. Par contre, les importations les plus denses relèvent seulement des entreprises de grande taille. La matrice des corrélations établie sur l'ensemble des variables fournit des cœfficients significatifs et voisins pour les PMI et les grandes entreprises. La sensibilité des exportations de produits à la taille est souvent importante quoique inégale. La corrélation des importations à la taille est bien nette pour tous les produits industriels.

Tout semble indiquer que les PMA ont un horizon marchand au mieux hexagonal. Les grandes entreprises pensent leur marché mais aussi leur approvisionnement au niveau mondial. Les PMI, quant à elles, envisagent l'international seulement pour les débouchés. Leur approvisionnement paraît plutôt se faire sur le marché national. Puisque les PMI sont deux fois moins orientées vers le marché mondial que leurs homologues de grande taille, il est tentant de penser qu'elles s'approvisionnent également moins sur les marchés étrangers. La capacité d'exportation française est principalement portée par les grandes entreprises, une bonne partie des flux d'importation également. Évitons toutefois les conclusions hâtives.

L'importation de produits, en particulier les biens d'équipement professionnel, les pièces détachées et les produits semi-finis passe majoritairement par des grossistes auprès desquels se fournissent les PMI. Seules les grandes entreprises, grâce à leur taille, peuvent court-circuiter ces réseaux de distribution. De plus, elles appartiennent fréquemment à des groupes multinationaux, leurs échanges commerciaux ne sont pas totalement libres. Ils obéissent aux principes établis par le siège social qui régit les relations entre les filiales situées dans différents pays. Les échanges des grandes entreprises correspondent souvent à du commerce intrafirme. Alors que les PMI sont rarement répertoriées comme telles, les grandes entreprises apparaissent donc comme importatrices directes. 
La taille, l'origine des capitaux et l'organisation de l'entreprise jouent un rôle discriminant dans la maîtrise des différentes opérations de la chaîne export (Léo, Monnoyer-Longe et Philippe, 1991). Ainsi, le profil des PME insérées dans les structures informationnelles, incontournables à l'exportation, possède des traits identiques à ceux du type PMI proposé ici. Cette conjonction d'éléments tend à confirmer que les PMI jouent un rôle actif dans les exportations. Mais ce que l'approche médiatique ou monographique peut mettre en exergue ne transparaît pas dans les données statistiques, en partie à cause de l'agrégation des PMI avec les PMA dans le vaste ensemble des PME. Le rôle des PMI à l'exportation renvoie d'abord à la relative banalisation, tant économique que culturelle, du fait international. La densification de l'espace économique (transports, télécommunications,...) a certainement rendu plus proches des marchés jugés jusque-là éloignés des régions d'implantation des PMI. Cette nouvelle proximité se retrouve dans les échanges internationaux des régions.

\subsection{Une sous-traitance régionale des échanges extérieurs}

Dans l'ensemble, trois groupes distincts forment la structure régionale du commerce extérieur français (voir figure 1). Les régions semblent ordonnées en fonction de l'intensité et non du contenu de leur échanges. De faibles échanges sur certaines catégories de produits ne sont pas compensés par des échanges importants sur d'autres catégories. Ce constat se vérifie aussi pour les modalités moyennes ou fortes. Les régions à exportations intenses sont également les régions à importations fortes. Il existe donc une liaison étroite entre le niveau des exportations et des importations.

Par contre, les régions frontalières sont plus extraverties que les autres. Plus précisément, la présence d'une frontière terrestre induit une nette densité des échanges extérieurs. Les relations économiques ont toujours passé outre l'existence de frontières administratives. La division du travail et la polarisation de l'espace marchand ont développé des zones attractives pour leur périphérie, que celle-ci soit de même nationalité ou étrangère. Mais ce qui n'est qu'échanges interrégionaux dans le premier cas devient import-export dans le second. Ainsi, le statut frontalier a une influence sur le niveau des échanges extérieurs des régions.

L'ouverture imminente du marché unique va accentuer le phénomène. La cartographie des points forts et des points faibles des échanges communautaires des régions françaises repose sur les bassins d'emploi industrialisés, principalement situés aux frontières (Laurencin, 1989). Ces bassins ne sont pas pour autant les plus vulnérables à l'unification du grand marché grâce à leur tradition industrielle éprouvée et à leur haut degré de concentration. L'abolition des frontières internes à la CEE va plutôt creuser l'écart entre les régions impliquées et les régions indifférentes aux échanges communautaires. 


\section{FIGURE 1}

Résultats régionalisés du commerce extérieur, intensité des exportations et importations selon la taille des entreprises, 1989.

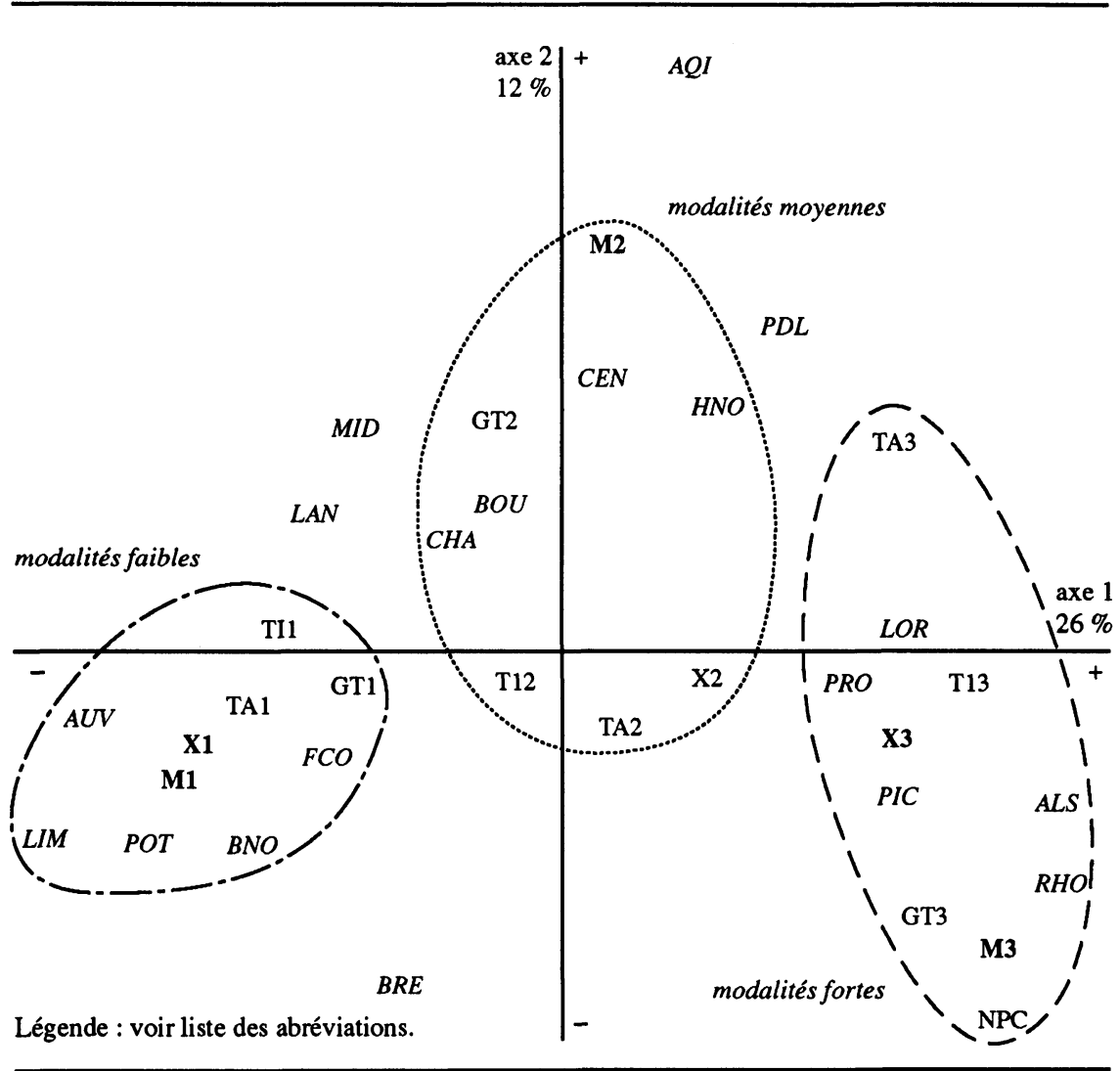

NPC : Nord Pas de Calais

PIC: Picardie

CEN : Centre

HNO : Haute Normandie

BNO : Basse Normandie

BRE : Bretagne

PDL : Pays de la Loire

POT : Poitou Charente

LIM : Limousin

AQI : Aquitaine

MID : Midi Pyrénées

CHA : Champagne Ardenne

LOR : Lorraine

ALS : Alsace

FCO : Franche Comté
LAN : Languedoc Roussillon

PRO : Provence Alpes Côtes d'Azur

TA1 : PME type artisanal, modalités faibles

TA2 : PME type artisanal, modalités moyennes

TA3 : PME type artisanal, modalités fortes

TI1 : PME type artisanal, modalités faibles

TI2 : PME type artisanal, modalités moyennes

TI3 : PME type artisanal, modalités fortes

GT1 : Grandes entreprises, modalités faibles

GT2 : Grandes entreprises, modalités moyennes

GT3 : Grandes entreprises, modalités fortes

X1 : Exportations, modalités faibles

X2 : Exportations, modalités moyennes

X3 : Exportations, modalités fortes 
BOU : Bourgogne

AUV : Auvergne

RHP : Rhône Alpes
M1 : Importations, modalités faibles

M2 : Importations, modalités moyennes

M3 : Importations, modalités fortes.

Il ne suffit pas qu'une région ait une frontière pour qu'elle voit se développer des échanges commerciaux typés qui la distinguent de ses voisines. Les résultats de l'analyse factorielle des correspondances menée sur la répartition régionale des échanges par pays permettent de faire apparaître des déterminants supplémentaires significatifs. On observe des oppositions sensibles dans l'orientation des flux régionaux du commerce extérieur. On note également des relations de proximité région/pays déjà mises en évidence de façon ponctuelle (Maillat, 1990).

Dans l'ensemble, les flux régionaux d'échanges avec les pays du Sud ou d'outre-mer se distinguent des flux d'échanges avec les pays d'Europe du Nord sur un axe Sud/Est et sur un axe Nord/Est. Les régions dont les échanges sont importants avec l'Europe du Nord-Est (République fédérale d'Allemagne) ont des relations marginales avec les pays du Sud (Maghreb, Espagne, Italie) et réciproquement. Un commerce intense avec les pays de second plan en Europe (Belgique, Pays-Bas) implique pour les régions des échanges faibles avec les grands pays industrialisés (République Fédérale d'Allemagne, États-Unis).

À ce constat s'ajoute une nette opposition des régions Nord face aux régions Sud. Les régions centrales sont moins distinctes. On relève des relations étroites entre les régions et leurs pays limitrophes respectifs. De ce point de vue, le commerce extérieur français ressemble fort à un « commerce de proximité » dans le sens où l'entend la typologie des canaux de distribution. Le commerce extérieur français avec les pays de proximité immédiate donne ainsi l'apparence d'être sous-traité aux régions limitrophes. L'observation, quoique atténuée, reste valable pour les pays plus éloignés.

Les importations sont plus différenciées que les exportations. On peut en déduire que les premières sont bien définies par région, ce qui est moins le cas des secondes. Les exportations ne sont pas indistinctes, mais il semble y avoir moins de relation privilégiée d'exportation région/pays. Le Traité de Rome a sans doute conduit à rendre plus homogènes les contours d'un marché européen sur lequel les entreprises françaises se placent indifféremment de leur localisation et de celle de leurs clients. Cela vaut surtout pour l'Europe du Nord, déjà ancienne.

Mais l'indifférenciation des échanges devient un handicap lorsque les autres pays ne partagent pas cette caractéristique. La difficulté d'établissement des relations avec l'étranger est d'intensité différente selon qu'il s'agit de la recherche d'un fournisseur ou de la prospection d'un client. On comprend dès 
lors pourquoi les régions françaises sont mieux positionnées par leurs importations que par leurs exportations. Le passé colonial de la France accentue la tendance, car il en résulte encore des relations privilégiées. L'approvisionnement de l'ancienne métropole en produits de base est loin d'être compensé par les possibilités d'exportation vers les pays concernés, compte tenu de la solvabilité de leur marché respectif. Ce commerce d'importation passe principalement par les régions méridionales.

De façon générale, l'effet de proximité est indéniable. Il n'obéit pas au seul critère de localisation géographique. Il correspond aussi à une proximité économique. Les activités exercées de part et d'autre de la frontière doivent être en correspondance pour générer des flux notoires. Une telle liaison relève parfois des relations amont/aval prévalant dans les filières de production existantes. Elle renvoie aussi à des pratiques diverses comme la sous-traitance de spécialité ou l'externalisation d'activités secondaires, en particulier de services: toutes choses concourant de façon périphérique à l'élaboration du produit fini, objet du mouvement d'exportation ou d'importation.

\section{D'une proximité de lieu à une proximité de délai}

\subsection{Une proximité économique de complémentarité}

Des modes de vie peu différents peuvent privilégier l'identité de produits des deux côtés de la frontière, selon la déclinaison des gammes pour la consommation finale. Pour la production, la similitude des activités peut conduire à des relations étroites de partenariat d'entreprises ou de sous-traitance, quand ce ne sont pas des liaisons financières impliquant des échanges intrafirme. La position géographique a sans doute favorisé des flux transfrontaliers anciens. Sur ce terreau, les entreprises ont su tisser un réseau dense d'échanges qui a accentué à son tour une proximite économique aujourd'hui déterminante.

L'effet de proximité se vérifie dans les deux sens. Les exportations par pays et par région sont bien corrélées aux importations pour les mêmes pays. Cela s'explique d'abord par le niveau d'activité de chaque région. Les importations sont sensibles aux besoins d'approvisionnement, les exportations relèvent des capacités d'expédition. Si bien que la densité des échanges extérieurs reflète l'importance de l'activité régionale. À cela s'ajoutent la complémentarité des procédés, la spécialisation sur des créneaux particuliers d'activité et la maîtrise de savoir-faire complexes qui aboutissent à lier exportations et importations. Le contenu en importation des exportations de la France approche $23 \%$ en 1989 (Barge et Moyne, 1991). Les échanges croisés franco-allemands grâce à Airbus Industrie sont exemplaires à cet égard. 
On ne peut cependant pas établir de corrélation exclusive entre exportations et importations pour chaque pays. Le commerce extérieur de la France échappe pour une part aux déterminants hexagonaux ou régionaux. Il est influencé par la position relative des pays concurrents ou partenaires (fournisseurs ou clients), voire par leur éventuelle spécialisation dans des produits d'approvisionnement pour les entreprises françaises. D'autre part, la France, pays intermédiaire dans la division internationale du travail, développe de façon sensible des flux croisés de mêmes produits, mais d'origine et de destination différentes selon le contenu en capital et en travail de la valeur ajoutée des produits (Henner, 1989).

En définitive, l'effet de proximité est assujetti à la spécialisation productive des régions. La nomenclature disponible ici n'a pas permis d'établir d'indice de spécialisation par région suffisamment fiable pour être significatif. Les indicateurs de spécialisation du commerce extérieur sont particulièrement sensibles au degré de désagrégation des catégories de produits (De Montbrial, 1990). Au niveau des grandes branches, les exportations et les importations de produits de même catégorie sont fréquentes, sans qu'on puisse établir s'il s'agit là d'échanges de produits identiques ou d'échanges intrabranche. De plus, la spécialisation des échanges de produits ne contient pas, en elle-même, d'information sur la spécialisation par procédés ou sur la cohérence par filière, exception faite des indications sur la concentration par branche des exportations et des importations. Le commerce extérieur français possède pour certains les caractéristiques d'un "bazar » où l'on peut trouver toutes sortes de produits (Le Masne, 1989). La répartition régionale des activités, nécessairement inégale, risque de forcer le trait.

Peu de spécialisations régionales marquantes apparaissent par grande catégorie de produits exportés ou importés. L'échelon régional sur lequel porte la mesure de la spécialisation en est sans doute la cause, car les spécialisations d'activités repérables au niveau départemental n'apparaissent pas toujours au niveau régional (INSEE, 1990). La répartition régionale des exportations et celle des importations sont corrélées pour les produits de complémentarité. Pour les produits de la métallurgie, de la chimie, pour les équipements professionnels et les biens de consommation, les cœfficients varient de 0,901 à 0,965 . Il s'agit de produits pour lesquels les relations de complémentarité sont fortes, soit en raison des formes de la division du travail pour ces produits (localisation typée amontaval dans la chimie et la parachimie, flux de perfectionnement dans le textilehabillement, délocalisation dans des filiales ateliers pour l'automobile ou l'électronique...), soit parce que les compléments de gamme par import-export sont fréquents. Pour ces produits, les régions les plus exportatrices sont souvent les plus importatrices, confirmant ainsi la complémentarité évoquée (Renard, 1989). 
Par contre, les produits pour lesquels les fabricants français et étrangers sont en concurrence directe sur le marché n'ont pas de corrélation entre flux régionaux d'exportations et d'importations. Ce sont des produits destinés avant tout à la consommation finale: denrées agricoles, produits agro-alimentaires, biens d'équipement ménager, automobiles. Dans ces cas, les régions exportatrices se distinguent des régions importatrices. Les fabricants français n'organisent évidemment pas l'arrivée de produits concurrents étrangers sur leur marché. Les réseaux d'importation sont donc localisés sur les lieux d'arrivage ou de grande consommation, au gré des créations d'entreprise ou des rachats, mais en toute indépendance de la localisation des entreprises françaises concurrentes, plus précisément des plus grandes. Il convient en effet de considérer le critère de la taille des entreprises, car les flux régionaux du commerce extérieur n'y sont pas insensibles.

À cet égard, la Haute-Normandie occupe une place à part. Cette région a la plus forte propension à échanger. Cette position de tête ne correspond pas à une spécialisation particulière par produits exportés ou importés. On exporte et on importe beaucoup dans cette région, mais sans produit de préférence. La Haute-Normandie est aussi la région qui accueille le plus de PME de type industriel. Les PMI y occupent une position déterminante avec près de $49 \%$ des effectifs du champ étudié pour la région. Cette présence est peut-être à l'origine de l'absence de spécialisation par activité. Mais elle explique mieux la propension à échanger de la région par la souplesse des PMI. Les délais de fabrication constituent en effet un facteur décisif sur tous les marchés.

Une telle singularité régionale provient également de l'importante plateforme du port du Havre. Le commerce de transit n'est pourtant pas répertorié par les Douanes et la capacité de production, certes importante, de la HauteNormandie n'explique pas tout. Reste à envisager le rôle des opérateurs du transport de marchandises qui peuvent générer sur place une valeur ajoutée particulière, hors transit et négoce, grâce au reconditionnement des produits. On sait que ces opérateurs sont de taille modeste : $98,1 \%$ des entreprises de transport routier de marchandises ont moins de 50 salariés et $78,1 \%$ en ont 5 au plus (Braibant, 1991). Le rôle premier de la Haute-Normandie dans les échanges extérieurs résulte donc d'activités, industrielles ou non, où les PME de type industriel sont très présentes. De manière plus générale, ce sont sans doute les nouvelles formes de sous-traitance internationale qui expliquent le mieux l'activité des PMI à l'exportation ${ }^{3}$.

3 L'état des statistiques douanières ne permet pas de dégager directement ces nouvelles formes; il faut s'appuyer sur une conjecture plus large. Aussi les références deviennent-elles plus fréquentes, car plus factuelles pour la fin de ce texte. 


\subsection{Une proximité de délai par flexibilité}

Après le redéploiement et la délocalisation, la décennie 1990 parait être celle de la sous-traitance internationale (STI). Les opérations de montage, assemblage de pièces détachées et transformation de produits semi-finis pour compte d'autrui sont en plein développement. Lorsque les entreprises contractantes appartiennent à des pays différents, il s'agit de STI. L'essentiel de la croissance récente des échanges communautaires provient de la STI, alors que les flux traditionnels subissent un léger tassement (Charbit, Ravix et Romani, 1991).

La STI des entreprises françaises, longtemps localisée dans le tiers monde, opère aujourd'hui une relocalisation relative en Europe du Sud (Mouhoud, 1990). Les importations intracommunautaires après perfectionnement passif (preneur d'ordre à l'étranger) sont cinq fois plus importantes que les importations (après p. p.) extracommunautaires. Cela peut expliquer un mouvement d'importation d'Espagne et d'Italie plus fort que du Maghreb. Mais l'ensemble de ces importations est quatre fois plus faible que les exportations françaises après perfectionnement actif (preneur d'ordre en France). Dans ce cas, les exportations extra-CEE sont majoritaires face aux exportations intracommunautaires.

Contrairement à une idée reçue, le marché de la STI s'ouvre donc plus rapidement aux PMI françaises qu'à leurs homologues du tiers monde. Ces dernières sont redoutables dans la sous-traitance de capacité, mais au prix d'une dépendance et d'une vulnérabilité extrêmes qui rappellent la situation des PMA en France. Les PMI sont rarement présentes sur les marchés pour ce qui est des produits. Cela ne semble pas rédhibitoire. Elles détiennent plutôt des technologies de procédé ou des savoir-faire particuliers qu'elles valorisent dans le cadre de la sous-traitance de spécialité (Brechet, 1990). Absentes des marchés internationaux, les PMI sont de plus en plus présentes dans les relations de proximité induites par la sous-traitance de perfectionnement actif. On comprend dès lors leur position privilégiée dans les flux régionalisés d'exportation.

La négociation du cahier des charges, le coût du visitage, l'entretien des équipements sont mieux maîtrisés à proximité ; d'autant plus que les tâches de fabrication semblent réalisées avec plus de soins, par des ouvriers somme toute plus qualifiés en Europe. Par contraste, l'importateur taïwanais a quelques fois fait faillite lorsque le conteneur de marchandises atteint son lieu de destination après 2 mois de trajet. L'exemple n'est pas anecdotique, puisque fréquemment relevé dans les secteurs des biens d'équipement ménagers et des biens de consommation courante. Il illustre les aléas d'une sous-traitance extrême, voire exotique. La relocalisation en Europe de la STI auparavant dévolue outre-mer paraît donc s'expliquer par des contraintes de contrôle de qualité et de délai de réaction. 
Le phénomène n'est pas réservé à la France. La STI se répartit en deux zones dans la CEE. La France, la Belgique, la République fédérale d'Allemagne et les Pays-Bas développent des liaisons circulaires de sous-traitance très fortes. Les autres pays de la CEE gravitent autour de cette zone centrale, entretenant avec elle des relations de sous-traitance bilatérales. La STI renvoie à une complémentarité centre/périphérie plutôt qu'à une opposition Nord/Sud. Un pays septentrional comme la Grande-Bretagne a un statut quasi identique à l'Espagne dans ce schéma européen de la sous-traitance. Le centre cumule les avantages de la proximité géographique des marchés, les économies d'échelles liées à la taille et à la densité du tissu industriel. La périphérie voit ses coûts de production faibles handicapés par les coûts de transaction, de contrôle de qualité et les frais de transport. À cela s'ajoutent des ressources humaines de faible qualification, une proportion plus élevée de secteurs sensibles et de petites entreprises à efficacité marginale. Les « marchés » de l'Europe ont un PIB par habitant inférieur à $70 \%$ de la moyenne communautaire.

Finalement, l'ordonnancement des échanges extérieurs des régions françaises obéit à la même structure concentrique. Les régions situées dans ou à proximité immédiate du noyau dur de la CEE voient leurs échanges se densifier. Les régions centrales et de la façade atlantique sont dans une toute autre configuration. Elles souffrent de périphéricité (Commission des Communautés Européennes, 1990). Paradoxalement, l'achèvement du maillage déjà dense des infrastructures de transport et de télécommunication aggrave les disparités. L'indifférenciation de l'espace aboutit à la polarisation et non à l'uniformisation du paysage économique. Avec des communications plus aisées, la densité du tissu industriel local et la proximité du marché deviennent des critères prépondérants de localisation (Tandeau 1991).

Les activités logistiques efficaces tendent à se généraliser. Cela signifie une meilleure maîtrise des flux de marchandises. L'espace, les distances n'ont plus les mêmes dimensions. La présence d'une plate-forme intermodale de chargement devient aussi importante que l'existence d'un site industriel notoire pour initier les échanges extérieurs d'une région. Le niveau des exportations ou des importations dépendant d'une telle plate-forme est d'autant plus croissant que les frais de transport diminuent dans les coûts logistiques. La concentration industrielle et commerciale diminue le nombre de points de liaison. La tendance au « zéro stock» multiplie les petites séries. La remontée du marché vers la production accroît les besoins en conditionnement, alotissement et autre étiquetage. Bref, la valeur ajoutée logistique découle du coût du kilomètre parcouru. Sa part dans la valeur totale d'un produit a doublé en moins de dix ans (Colin et Pache 1988).

Lorsque la gestion des stocks en flux tendu se développe dans les entreprises, les pratiques logistiques deviennent primordiales pour la circulation 
continue des marchandises. La fluidité des opérations passe par l'activité d'un nombre croissant d'entreprises spécialisées. La multiplication des opérateurs associée à la polarisation des activités implique en définitive que la proximité de délai supplante la proximité de lieu.

Dans ce cadre, l'improvisation ou l'approximation sont rarement efficaces. Les nouvelles formes de la sous-traitance et de l'externalisation multiplient autant qu'elles sélectionnent les partenaires pour chaque entreprise. La croissance du nombre d'intervenants provient de la spécialisation par métier des opérations jusque-là intégrées. Mais pour chaque type d'opération, on assiste à un resserrement du choix des interlocuteurs, sélectionnés selon des critères de qualité et de fiabilité du service rendu.

Pour chaque entreprise, la chaîne d'activité se complexifie et s'allonge. Le temps prend une dimension stratégique. En particulier, le délai de réaction de chacun des multiples opérateurs occupe une position centrale. Durée de conception et de mise au point des produits, temps de montage et d'assemblage, délai de livraison amont ou aval, rapidité des interventions de maintenance, temps de négociation commerciale, durée des opérations du S.A.V., crédit fournisseur... Et la liste n'est pas exhaustive.

D'un autre côté, les mutations des systèmes de transport et de télécommunication rendent l'espace plus accessible, plus indifférencié. La polarisation des activités sur le territoire s'accompagne d'une radialisation de l'espace. La distance n'est plus une métrique, les réponses nodales sont plus rapides (Bonnafous, 1990). La proximité de délai prend dès lors le pas sur la proximité de lieu. Le temps de réalisation de l'opération, par le partenaire, le sous-traitant ou le prestataire de service devient déterminant dans les relations interentreprises. Peu importe la distance à parcourir pour rendre effectives ces relations. Les équations de coûts s'écrivent désormais sous la contrainte majeure du temps.

Dans ce contexte, le délai de réaction dont peuvent faire preuve les PMI est décisif. Leur taille leur permet une souplesse notoire dans l'organisation de la production. Leur flexibilité, lorsqu'elle est fondée sur la qualification du personnel et l'investissement de productivité, rend compatibles qualité et compétitivité à court terme. A contrario, les avatars du circuit court du modèle du Sentier dans la confection (Montagne-Villette, 1990) ou, plus généralement, la démographie tourmentée des PMA (Viennet, 1990) sont là pour rappeler que les contraintes de temps renvoient à une flexibilité bien comprise. Les PME de type industriel paraissent ainsi mieux disposées pour répondre aux exigences de la proximité de délai.

Cela étant, il ne faut pas oublier la prégnance du territoire dans les relations de proximité. C'est ce qu'illustre la réussite du modèle adriatique (appel- 
lation plus poétique sans doute que celle de districts marshalliens), par référence à cette contrée italienne où des PME, organisées en réseaux territoriaux denses, ont obtenu des percées remarquables (Moynot, 1988). Les "success stories » italiennes prouvent d'ailleurs que les relations de proximité ne se déplacent pas simplement de l'espace vers le temps. Au-delà de la diversité des modèles explicatifs de cette réussite (Savi, 1989), un invariant apparaît : ce type d'organisation de PME n'est pas la résultante du hasard.

On observe que l'horizontalité des réseaux comme des districts n'est souvent qu'apparente. Les relations interentreprises renvoient toutes à une hiérarchie formelle ou informelle mais de toute façon présente. Les entreprises «chef de file " des réseaux peuvent éventuellement être de petite dimension, mais elles utilisent toujours des fournisseurs subalternes ou des entreprises imitatrices. Elles ont un rôle commercial particulièrement actif et une politique de RechercheDéveloppement explicite. D'un autre côté, les firmes présentes dans les phases désintégrées du cycle de production sont des micro-entreprises, souvent souscontractantes en relation avec un unique donneur d'ordre. Leur compétence repose fréquemment sur un savoir-faire manuel et rarement sur la maîtrise d'un procédé capitalistique.

La typologie, certes rudimentaire, des PME proposée dans cette étude éclaire cette hiérarchie d'un jour nouveau. Le portrait des entreprises " chef de file » ressemble fort à celui des PME de type industriel, tandis que le profil des micro-entreprises rappelle plutôt les PME de type artisanal. La similitude est double, il ne peut s'agir d'une pure coïncidence. La dynamique particulière des réseaux de PME dans les districts industriels résulte, en dernière instance, des combinaisons établies entre proximité de lieu et proximité de délai. La densité du territoire en entreprises similaires ou complémentaires rend possible une rapidité de réaction tout comme des économies d'échelle ou de champ. Dans ce cas, la proximité de délai résulte en partie de la proximité de lieu. L'opposition de termes renvoie à une complémentarité des relations et non à une substitution de tendance. L'existence de réseaux intersites où sont combinées spécialisation technique et polarisation territoriale (Gilly, 1991), tend à prouver que la proximité de délai est parfois assujettie à la proximité de lieu. En ce sens, la distinction PMI/PMA révèle beaucoup plus une conjonction de pratiques pour chaque catégorie, qu'un dualisme factuel bien établi.

\section{Conclusion}

Au terme de cette étude, nous pouvons observer que le rôle actif des PME dans le commerce extérieur n'est perceptible qu'après décomposition de la catégorie apparemment homogène des PME en PMA et PMI. L'intensité des échanges 
extérieurs augmente avec la taille des entreprises. Les PME de type artisanal semblent hors marché. Les grandes entreprises sont nettement extraverties, légèrement plus à l'import qu'à l'export. Par contre, les PME de type industriel sont mieux placées à l'export qu'à l'import. Cela s'explique par l'insertion particulière des PMI dans le territoire économique. Au niveau régional, les échanges extérieurs subissent un effet frontalier. Il existe une relation étroite entre les régions et leurs pays limitrophes respectifs. Elle correspond plus à une complémentarité économique qu'à la seule position géographique. Les PMI sont partie prenante dans ce véritable commerce de proximité. Discrètes sur les marchés mondiaux, elles sont de plus en plus actives dans la sous-traitance internationale de perfectionnement qui porte l'essentiel de la croissance actuelle des échanges. La multiplication des opérateurs, associée à la polarisation de l'espace implique en définitive que la proximité de délai supplante la proximité de lieu. Les PMI sont concernées dans les deux cas.

Aujourd'hui, le développement des stratégies de grappes repose sur des relations horizontales entre les entreprises. La valorisation des technologies génériques est un des principaux facteurs expliquant l'apparition des réseaux de firmes (Zimmerman, 1989). Ces nouvelles pratiques sont éloignées de la soustraitance traditionnelle. Les liaisons de partenariat, de coopération supposent la fiabilité des interlocuteurs, sinon leur durabilité. Ces perspectives mettent encore plus les PMA hors marché. La tendance à la création de PMA filiales de groupes laisse cependant penser que ce type d'entreprise va être de plus en plus sollicité dans l'ajustement fin de la nouveauté.

D'un autre côté, les caractéristiques des PMI peuvent pleinement cadrer avec ces nouveaux schémas de comportement. Elles allient l'adaptabilité et la flexibilité redoutables des petites entités à la rigueur et à l'efficacité bien réelles des grandes structures. À l'avenir, le critère de la qualité des partenaires prévaudra probablement sur celui de leur nationalité. En ce sens, l'abolition des frontières internes de la CEE risque d'accentuer la polarisation de proximité ou de taille des flux régionalisés du commerce extérieur français.

Immergées dans le tissu économique de leur territoire d'implantation, en prise directe avec les décideurs locaux, jouant la carte de la spécialité et du savoir-faire technologiques, les PMI sont au cœur de la proximité de lieu. En phase avec un marché de plus en plus segmenté en niches étroites, disposant d'une productivité incomparable pour les petites séries de qualité, leur taille leur confère la souplesse indispensable pour aborder dès maintenant la fluidité des échanges. Les PME de type industriel semblent bien être les entreprises dont la proximité de délai a besoin. Pour leur part, les PMI des régions frontalières devraient pouvoir sans dommage conjuguer les avantages encore présents d'une proximité de lieu et les atouts décisifs de la proximité de délai. 
Le passage du discours prédictif à la vraisemblance logique nécessite bien évidemment de dépasser les limites trop étroites de cet article. En particulier, il convient d'affiner et de tester de façon plus rigoureuse la typologie des PME proposée ici. En outre, il paraît souhaitable d'aborder des nomenclatures plus détaillées et si possible d'élargir le domaine d'investigation aux flux régionalisés du commerce extérieur des principaux pays partenaires de la France.

\section{Sources}

Les entreprises. Le fichier Sirène de l'INSEE fournit la répartition selon les régions des effectifs des entreprises au 12 janvier 1990, en 15 secteurs d'activité (NAP 15) et par taille d'entreprise. La localisation en Île-de-France de la plupart des sièges sociaux des grandes entreprises, alors qu'elles ont très fréquemment des établissements en province, aboutit à une surreprésentation de ladite région dans cette catégorie de taille. Les entreprises de 500 salariés et plus y occupent $51 \%$ des effectifs industriels et agricoles contre $13 \%$ à $31 \%$ dans les autres régions. On peut admettre que la localisation francilienne des grands sièges sociaux affecte de façon indifférenciée la majorité des régions. Les régions rurales sont épargnées à cause de leur faible industrialisation. Les grosses régions industrielles semblent aussi moins touchées. En raison de leur importance et de leur éloignement de la capitale, elles possèdent leur propre hinterland. L'élimination de l'île-de-France du champ d'investigation permet d'atténuer la déformation de la répartition spatiale de l'activité dans la mesure où cette région exerce une attraction relativement homogène sur les autres. La répartition des effectifs par établissement éviterait sans doute mieux ce biais. Mais l'interprétation de l'incidence des tailles sur les échanges extérieurs deviendrait impossible. La localisation réalisée par les Douanes dépend de l'établissement d'origine ou d'arrivée des marchandises. Cependant, une importation effectuée dans un petit établissement peut relever d'une grande entreprise, comme d'une PME. Si la probabilité de posséder plusieurs établissements augmente avec la taille de l'entreprise, il n'y a pas de relation certaine entre la taille de l'entreprise et celle de ses établissements. Il existe cependant une bonne corrélation entre la répartition régionale des effectifs des entreprises et celle des effectifs des établissements, activité par activité, taille par taille : les cofficients varient de 0,703 à 0,999 . Retenir le niveau de l'entreprise pour représenter la répartition régionale des activités par taille n'aboutit pas à une perte considérable d'information. Par contre, l'analyse gagne en signification, car les entreprises opèrent sur le marché international non leurs établissements. Sont pris en compte ici les 5 secteurs d'activité de production (hors énergie) se rapprochant le plus des catégories de produits des Douanes. À ce niveau très agrégé de la nomenclature d'activité, la correspondance branche/produit, préférable, n'est pas trop 
éloignée de celle retenue. Ce même niveau ne permet pas de considérer dans cette étude le rôle pourtant avéré des opérateurs tertiaires dans les échanges extérieurs de produits. Sont donc exclus les secteurs tertiaires: du commerce aux services non marchands.

Le commerce extérieur. L'étude porte sur les résultats régionalisés des statistiques du commerce extérieur français de l'année 1989 établis par les Douanes à partir de la banque de données Béatrice. Les exportations sont évaluées $\mathrm{FAB}$, les importations sont comptées CAF. L'île-de-France et la Corse, à cause de valeurs extrêmes, respectivement $26 \%$ et $0,02 \%$ des échanges extérieurs, n'ont pas été retenues. L'analyse porte sur 20 régions. Il n'est nulle part question des flux interrégionaux d'approvisionnement ou d'expédition; seules les exportations et les importations sont considérées ici. Pour les échanges communautaires, l'Irlande, le Danemark, la Grèce et le Portugal représentant $0,4 \%$ à $1 \%$ des échanges, sont exclus du champ. Pour le reste du monde, seules les valeurs significatives ( $>=2 \%$ des échanges) sont étudiées. Les USA, l'Europe Occidentale, l'Europe de l'Est et le Maghreb sont donc pris en compte. Le reste est soit marginal, soit insuffisamment localisé pour avoir un sens. Ont été retenus au total 7 pays et 3 zones assimilées à des pays. Cet ensemble représente près de $80 \%$ du commerce extérieur de la France. Par produit, 9 catégories sont examinées. Outre le matériel militaire, non compté par les Douanes, sont éliminés du champ les matières premières minérales et les divers ( $<1 \%$ chacun), ainsi que les échanges de produits énergétiques, circonscrits à 5 régions et dont les déterminants échappent à la problématique posée ici.

Les outils statistiques. L'analyse des données est conduite en coupe transversale. L'élaboration de séries chronologiques n'est envisageable que pour les deux dernières années. Les Douanes ont mis en œuvre en 1988 une nouvelle méthode de classification par produit. De plus, la dimension temporelle est ici secondaire. La compétitivité-prix et l'état de la demande sont sensibles aux aléas temporels. Ce n'est pas le cas de la localisation de l'offre, exprimée en potentiel d'expédition ou en besoins d'approvisionnement. Les outils d'investigation sont fonction de ces données. Avec peu d'individus observés (20 régions) et des caractéristiques nombreuses et différentes pour chaque observation (tailles, secteurs, pays, produits, exportations, importations), l'analyse des données semble singulièrement appropriée pour ce genre d'étude. En particulier, l'analyse factorielle des correspondances est indiquée pour dégager oppositions et similitudes entre les régions ou entre variables. L'analyse factorielle des correspondances est établie à l'aide du logiciel Statitcf (Dervin, 1990) de même que la matrice des corrélations portant sur les variables des tableaux. Le matériau statistique de cet article à fait l'objet d'une communication au colloque international "le rôle des PME dans l'économie des régions transfrontalières " à Mulhouse les 21 et 22 mai 1991. 


\section{Bibliographie}

AMAR, M. et B. CREPON (1990), «Les deux visages de la concentration industrielle : efficacité et rente de situation », INSEE, Économie et Statistique, $n^{\circ} 229$, février, p. 5-19.

Banon, C. et M. Delbreil (1989), «Les entreprises françaises face au développement de leurs exportations », Banque de France, Paris, Centrale des Bilans, mars, p. 5.

BARGE, M. et V. MoYNE (1991), "Quelles utilisations pour nos importations ? », INSEE Première, $\mathrm{n}^{\circ} 120$, janvier, p. 4.

BEAU, D. (1990), «Les influences de l'appartenance à un groupe sur les structures et les résultats des entreprises industrielles françaises », Banque de France, Paris, Centrale des Bilans, mai, p. 20.

BERTHIER, C. et B. SAVOYe (1990), «La sous-traitance des petites entreprises industrielles en 1988 », INSEE Première, $\mathrm{n}^{\circ}$ 95, juillet, p. 2.

Biales, C. et G. Rolland (1989), «Les PME : atouts et handicaps », INSEE/CNDP, Ecoflash, n $^{\circ} 40$, juin, p. 3.

BonNafous, A. (1990), « Mutation des systèmes de transport et radialisation de l'espace », Revue d'Économie Régionale et Urbaine, ${ }^{\circ} 2$, p. 307-315.

BraIBANT, M. (1991), «Le transport routier de marchandises à la veille de l'échéance européenne », INSEE, Économie et Statistique, n 239, janvier, p. 3-17.

BRECHET, J.P. (1990), «Les savoir-faire industriels des PMI », Économies et Sociétés, série Sciences de Gestion, $n^{\circ} 15$, mai, p. 39-58.

Bricout, J.L. (1991), «Exporter : une question de taille », INSEE, Économie et Statistique, $\mathrm{n}^{\circ} 244$, juin, p. 25-33.

Bucallie, A. et B. Costa De Beauregard (1987), PMI, enjeux régionaux et internationaux, Paris, Économica, p. 34.

Charbit, C., J.T. Ravix et P.M. Romani (1991), « Sous-traitance et intégration industrielle européenne », Revue d'Économie Industrielle, $\mathrm{n}^{\circ} 55,1^{\circ}$ trimestre, p. 178 189.

Colin, J. et G. PACHE (1988), « La logistique de distribution » Paris, Chotard \& Associés Éditeurs, p. 183.

Commission des Communautés Européennes (1990), «L'impact régional de l'union économique et monétaire », Économie européenne $n^{\circ} 44$, Bruxelles, octobre, chap. 9.

De Montbrial, T. (1990), (sous la direction de), Ramses 1991, Paris, Dunod, p. 125.

DERVIN, C. (1990), « Comment interpréter les résultats d'une analyse factorielle des correspondances ? ", Paris, ITCF, mars, p. 7.

Di RuZzA, R. (1988), «Éléments d'épistémologie pour économistes », Grenoble, Presses Universitaires de Grenoble, p. 95.

GILly, J.P. (1991), «Dynamique territoriale des activités de l'espace » dans l'Europe industrielle: horizon 93, tome 2, Notes et Études Documentaires, $\mathrm{n}^{\circ} 4932-33$, juillet, p. 101-126.

Gollac, M. (1989), «Les dimensions de l'organisation du travail », INSEE, Économie et Statistique, $\mathrm{n}^{\circ} 224$, septembre, p. $27-44$. 
GuICHARD, J.P. (1987), «L'industrie, le régional et l'urbain », dans Industries et régions, ADEFI GRECO, Paris, Économica, p. 35.

HenneR, H.F. (1989), Commerce international, Paris, Montchrestien, p. 148.

Hy, M., B. Lassaut et F. Nicolas (1989), « Concurrence entre les formes industrielles et artisanales de production-distribution des biens alimentaires », Économies et Sociétés, série Progrès et Agriculture, $\mathrm{n}^{\circ}$ 20, juillet, p. 197-216.

INSEE, (1990), La France et ses régions, Paris, INSEE, p. 13.

JULIEN, P.A. (1990), «Vers une typologie multicritère des PME ", Revue Internationale $P M E$, vol. $3, n^{\circ} 3-4$, p. $411-425$.

LAURENCIN, J.P. (1989), «L'impact sectoriel et régional du grand marché : le cas de l'industrie française ", Revue d'Économie Industrielle, $\mathrm{n}^{\circ} 49,3^{\mathrm{e}}$ trimestre, p. 67-92.

LE MASNE, P. (1989), «La structure des échanges extérieurs dans une approche entréessorties : la France et ses voisins européens ", Revue d'Économie Politique, 99, $\mathrm{n}^{\circ}$ 6, novembre-décembre, p. 835-853.

Py, L., M.C. Monnoyer-Longe et J. PhILIPPe (1991), « Métropoles régionales et PME : l'enjeu international », Revue d'Économie Régionale et Urbaine, ${ }^{\circ}$ 1, p. 41-70.

MaIllat, D. (1990), «Régions transfrontalières et Marché Européen Unique. Le cas de la région Franche-Comté/Suisse Romande », Revue d'Économie Régionale et Urbaine, $\mathrm{n}^{\circ} 1$, p. 117-135.

MALIVERNEY, J. (1991), "Les entreprises exportatrices : une concentration accrue ", INSEE, Économie et Statistique, $\mathrm{n}^{\circ} 244$, juin, p. 15-24.

MARChesnay, M. (1991), «De l'hypofirme à l'hypogroupe », Cahiers du Lerass, $\mathrm{n}^{\circ} 23$, mai, p. 33-49.

Montagne-Villette, S. (1990), Le Sentier : un espace ambigu, Paris, Masson, p. 45.

Mounoud, E.M. (1990), "Stratégies de délocalisation et comportement d'imitation ", Revue de l'IRES, $\mathrm{n}^{\circ}$ 3, printemps-été, p. 81-117.

Moynot, J.L. (1988), Produire la nouveauté, Paris, Économica, p. 38.

QuELENNEC, M. (1987), «La sous-traitance industrielle gagne du terrain », INSEE, Économie et Statistique, $\mathrm{n}^{\circ} 200$, juin, p. 27-42.

ReNARD, M.F. (1989), "Les régions françaises et la qualification régionale », Revue d'Économie Politique, 99, $\mathrm{n}^{\circ}$ 5, septembre-octobre, p. 744-761.

SAvI, F. (1989), «Italie du centre, Troisième Italie, NEC, origine d'une spécification et fonctionnement d'un système industriel basé uniquement sur les PME », Revue Internationale PME, vol. 2, $\mathrm{n}^{\text {os }} 2-3$, p. 145-158.

TANDEAU, B. (1991), «Stratégies de localisation : les entreprises étrangères en France » dans l'Europe industrielle : horizon 93, tome 1, Notes et Études Documentaires, $\mathrm{n}^{\circ}$ 4926, février, p. 109-113.

TeMPle, P. (1986), «Le processus d'innovation dans les entreprises », Économie Appliquée, tome $39, \mathrm{n}^{\circ} 3, \mathrm{p} .583-614$.

VIENNET, H. (1990), « Survivre : premier souci des jeunes entreprises », INSEE Première, $\mathrm{n}^{\circ} 110$, novembre, p. 4.

ZimmeRmanN, J.B. (1989), «Groupes industriels et grappes technologiques », Revue $d^{\prime}$ Économie Industrielle, $\mathrm{n}^{\circ} 47,1^{\mathrm{er}}$ trimestre, p. 89-102. 